\title{
Personality Correlates for Digital Concert Program Notes
}

\author{
Marko Tkalčič, Bruce Ferwerda, David Hauger, and Markus Schedl \\ Department of Computational Perception \\ Johannes Kepler University \\ Altenberger Strasse 69, Linz, Austria \\ \{marko.tkalcic, bruce.ferwerda, david .hauger, markus.schedl\} @jku.at \\ http://www.cp.jku.at
}

\begin{abstract}
In classical music concerts, the concert program notes are distributed to the audience in order to provide background information on the composer, piece and performer. So far, these have been printed documents composed mostly of text. With some delay, mobile devices are making their way also in the world of classical concerts, hence offering additional options for digital program notes comprising not only text but also images, video and audio. Furthermore, these digital program notes can be personalized. In this paper, we present the results of a user study that relates personal characteristics (personality and background musical knowledge) to preferences for digital program notes.
\end{abstract}

Keywords: classical music, digital program notes, personality

\section{Introduction}

Classical music has long resisted the temptation of introducing new devices, such as smartphones or tablets into the live concert experiences. However, things started to change recently. Two efforts are being made in this direction, one within the FP7 Phenicx project ${ }^{1}$ and the other by the Philadelphia Orchestra ${ }^{2}$, both of them developing a mobile application for supporting the live classical concert experience. Both of these applications feature the program notes in a digital form. Within the Phenicx project we are developing a system that would support personalized digital program notes based on the user profile. The personalization is going to be done of the level of the preferred multimedia type in the notes (music, text or audio) and its length. In order to develop the personalization method we first need to identify the relationships between the users' personal characteristics (in terms of personality and musical background) and the preferences for the supporting multimedia material in the digital program notes. In this paper, we present the results of a user study that was carried out in order to identify these relationships. To be more concrete, we addressed the following research questions

\footnotetext{
1 http://phenicx.upf.edu/

${ }^{2}$ https://www.philorch.org/introducing-livenote $\%$ E2\%84\%A2-nights\#/
} 
1. Do the user's preferences for the digital program notes correlate with the user's personality

2. Do the user's preferences for the digital program notes correlate with the user's musical background

\section{Related work}

Personality and learning styles. The purpose of the program notes is to provide additional information about the concert in terms of the historical background on the piece, the composer and the performer. Similar educational processes, outside the classical music live experience domain, have been investigated in terms of personalization. Research based on learning styles, as a subset of personality, has been done [6] and models for personalizing course materials have been proposed [3].

Personality and user preferences. Personality has been gaining popularity in research on personalized services. It has been used to alleviate the coldstart problem in recommender systems $[4,15]$. Research has been carried out to understand how personality relates to user preferences in multiple domains [1]. Special attention has been given to music, where strong relations have been found (i) between personality and genre preferences [14] (ii) between personality and the way people use music (e.g. rationally versus for mood regulation [2]) and (iii) between personality and the way users organize music [5]. However, to the best of our knowledge, there has been no research done on the relations between classical music and personality, except for the aforementioned genre preferences.

Implicit acquisition of personality. For a long time, the only way of assessing the user personality has been through extensive questionnaires, such as the Big-Five Inventory (BFI) [9]. There has been an increasing interest for detecting unobtrusively the user personality from various sources. Related work has been done especially on using social media (e.g. facebook or twitter) to infer the personality in the Five Factor Model (FFM) personality space $[7,13,10]$. These methods can be useful in scenarios when a user logs in an online system with her/his social media account.

\section{Usage Scenario}

Within the Phenicx project, we are developing technologies for improving the classical concert experience. One of the use cases we focus on is the digital program notes scenario, where automatically generated, tailor-made multimedia editorials are offered to prospective audience members, introducing the concert that will take place with the purpose of informing, educating and pre-engaging users, supporting concert anticipation [11]. The concrete scenario we foresee is a user (the attendee of a classical concert) having a mobile device (e.g. a smartphone or a tablet device) with a mobile application that supports the scenario. The user logs in the application with her/his social media account (e.g. twitter or facebook). Using some of the aforementioned methods for inferring the user's 
personality from the social media stream, the application can use this information to tailor the digital program notes to the user's needs. The strategy for personalizing the digital program notes is going to be based on the outcomes of the study we present in this paper.

\section{Materials and Methods}

We conducted a user study to collect the data needed. We gathered the participants through Amazon Mechanical Turk. In total we had 165 participants aged from 20 to 76 (mean 37, stdev 11), 93 females and 72 males. The participants first filled in a set of background questionnaires: a questionnaire about their musical background, a questionnaire about their musical preferences in general (according to 18 genres) and the Ten-items Personality Inventory (TIPI) questionnaire [8]. The participants were then shown various supporting multimedia content that could fit in an interactive digital program note. For the chosen classical music concert (the piece La Mer by the French composer Claude Debussy performed by the Royal Concertgebouw Orchestra) we prepared multimedia material (text, images and audio) for the composer, piece and performer. Furthermore, each pair (e.g. composer-text) was presented in a long and short version (for images and audio clips short meant one item and long meant several items). In total we presented the participants 14 combinations of content. For each content combination, the participants gave three ratings: (i) amount of consumption of the content (on the scale none/some/half/most/all), (ii) interestingness of the content (on a scale from 1 to 5) and (ii) novelty of the content (on a scale from 1 to 5$)$.

\section{Results}

As can be seen from Tab. 1, personality traits were correlated with the subjects' musical background. Openness and extraversion were positively significantly correlated to three variables, agreeableness had a negative significant correlation with two variables while conscientiousness had a positive correlation only with the attending classical concerts variable. Neuroticism was not significantly correlated with any of the musical background variables.

Personality also appears to be correlated with how much the subjects like classical music as a genre (see Tab. 2). Openness, extraversion and agreeableness have a positive significant correlation with liking classical music, while conscientiousness and neuroticism are not significantly correlated.

We observed several significant correlations among variables that can be useful in a personalized system. Personality did correlate with with the answers provided by the subjects on the consumption, interestingness and novelty of the multimedia material. For example, people who score high on openness, agreeableness, conscientiousness and extraversion tend to show a positive correlation with consumption, interestingness and novelty, hence meaning that they prefer to consume more of the material, find it interesting and novel. On the other 


\begin{tabular}{llll}
\hline personality trait & musical background variable & correlation & $\mathrm{p}$ value \\
\hline openness & listening to classical music & 0.26 & 0.0007 \\
& studying an instrument & 0.16 & 0.03 \\
& attending classical concerts & 0.19 & 0.01 \\
\hline extraversion & playing an instrument & 0.21 & 0.008 \\
& attending classical concerts & 0.3 & 0.00009 \\
& attending non-classical concerts & 0.24 & 0.002 \\
\hline agreeableness & listening to non-classical music & -0.22 & 0.005 \\
& studying an instrument & -0.23 & 0.003 \\
\hline conscientiousness & attending classical concerts & 0.17 & 0.02 \\
\hline
\end{tabular}

Table 1: Correlations between personality traits and musical background variables.

\begin{tabular}{lll}
\hline personality trait & correlation & $\mathrm{p}$-value \\
\hline openness & 0.17 & 0.03 \\
extraversion & 0.21 & 0.006 \\
agreeableness & 0.33 & 0.00001 \\
conscientiousness & 0.11 & 0.16 \\
neuroticism & -0.14 & 0.06
\end{tabular}

Table 2: Correlation between personality traits and the preferences for the classical music genre.

hand, subjects who score high on neuroticism tend to consume less of the material, find it less interesting or novel. Our data show no correlation or negative correlations for neuroticism and the observed answers (see Fig. 1). Similarly, the music background variables showed generally positive correlations with consumption, interestingness and novelty of the multimedia material.

\section{Conclusions and Future Work}

The analysis of our data showed that there are many significant correlations between personal characteristics (personality and musical background) and preferences for the supporting multimedia content. However, further analyses should be carried out to determine how these personal characteristics relate with various types of material (text/images/audio) or length. The most important step we need to take is to use machine learning techniques to predict the preferred type of digital program notes based on the users' personalities. We plan to use logistic regression for individual binary variables and multinomial logistic regression for individual Likert scale ratings. Furthermore, we plan to use a rule-based algorithm for inferring the preferred modality and length.

We are currently in the process of designing a new user study where we will have better control over the text variance. The text will be curated by musicologists and we will use different levels of complexity (in terms of vocabulary). 

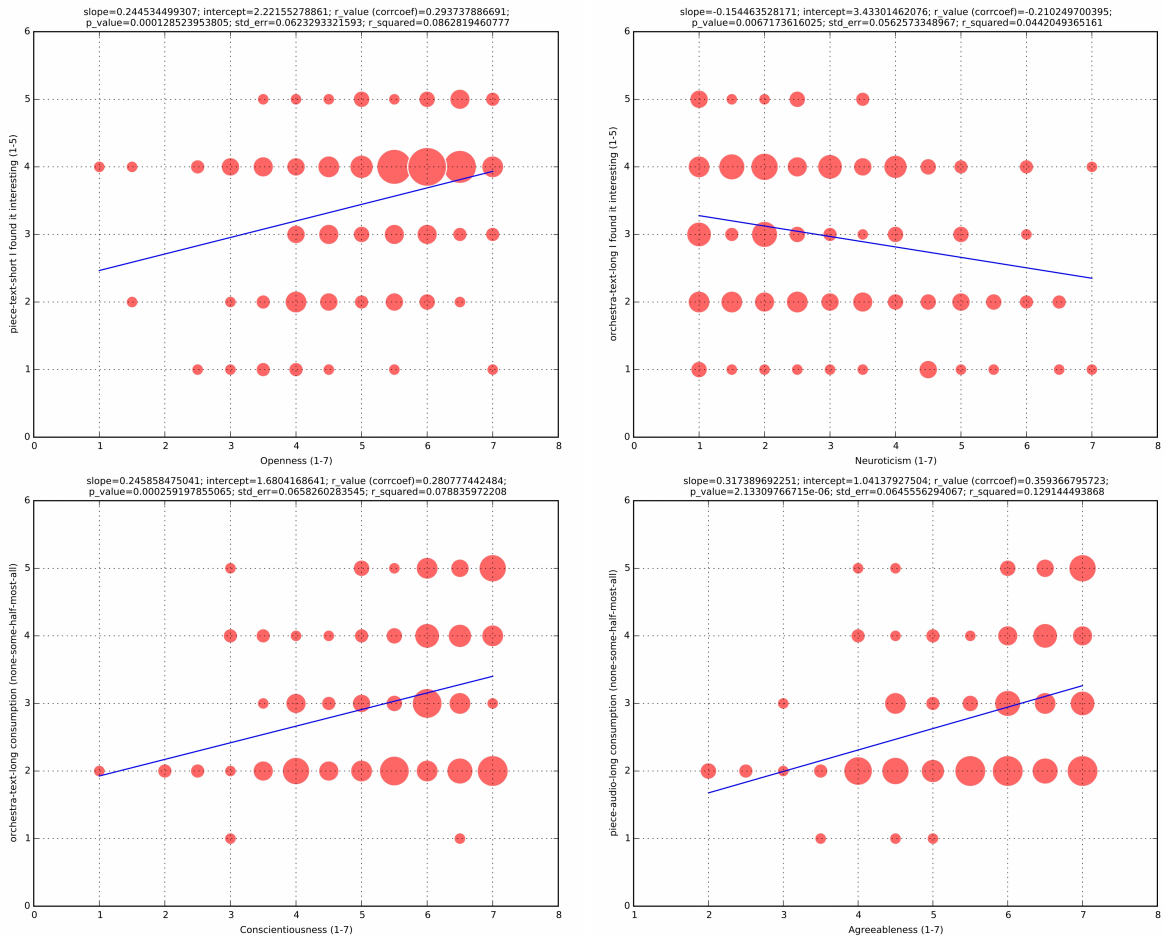

Fig. 1: Bubble plots with correlations between personality and content preferences. In the presented four cases all independent variables ( $\mathrm{x}$ axis) are on the scale from 1 to 7 , while the dependent (y axis) are on the scale from 1 to 5 . The top-left figure is the relation between openness and the preference for the short description of the piece (slope $b_{1}=0.24$, correlation $r=0.29$, p-value $p=0.0001$ ). The top-right figure is the relation between neuroticism and the preference for a long description about the orchestra $\left(b_{0}=0.15, r=-0.21, p=0.007\right)$. The bottom-left figure is the relation between conscientiousness and the degree of consumption of the long orchestra description $\left(b_{0}=0.25, r=0.28, p=0.003\right)$. The bottom-right figure is the relation between agreeableness and the degree of consumption of the long audio $\left(b_{0}=0.32, r=\right.$ $\left.0.36, p=2 \cdot 10^{-6}\right)$

Furthermore, we will use the Goldsmiths Music Sophistication Index (MSI) [12] to assess the musical background of the users (the MSI instrument was published after we already carried out the study presented here).

\section{Acknowledgement}

This research is supported by the European FP7/2007-2013 programme through the PHENICX project under grant agreement no. 601166 and by the Austrian Science Fund (FWF): P25655. 


\section{References}

1. Iván Cantador, Ignacio Fernández-tobías, and Alejandro Bellogín. Relating Personality Types with User Preferences in Multiple Entertainment Domains. EMPIRE 1st Workshop on "Emotions and Personality in Personalized Services", 10. June 2013, Rome, 2013.

2. Tomas Chamorro-Premuzic and Adrian Furnham. Personality and music: can traits explain how people use music in everyday life? British journal of psychology (London, England : 1953), 98(Pt 2):175-85, May 2007.

3. Moushir M. El-Bishouty, Ting-Wen Chang, Sabine Graf, and Nian-Shing Chen. Smart e-course recommender based on learning styles. Journal of Computers in Education, 1(1):99-111, March 2014.

4. Mehdi Elahi, Matthias Braunhofer, Francesco Ricci, and Marko Tkalcic. Personality-based active learning for collaborative filtering recommender systems. AI*IA 2013: Advances in Artificial Intelligence, pages 360-371, 2013.

5. Bruce Ferwerda, Emily Yang, Markus Schedl, and Marko Tkalčič. Personality Traits Predict Music Taxonomy Preference. In CHI 2015 Works-in-Progress, 2015.

6. Adrian Furnham. The big five versus the big four: the relationship between the Myers-Briggs Type Indicator (MBTI) and NEO-PI five factor model of personality. Personality and Individual Differences, 21(2):303-307, August 1996.

7. Jennifer Golbeck, Cristina Robles, Michon Edmondson, and Karen Turner. Predicting Personality from Twitter. In 2011 SocialCom and PASSAT conference Proceedings, pages 149-156. IEEE, October 2011.

8. Samuel D Gosling, Peter J Rentfrow, and William B Swann. A very brief measure of the Big-Five personality domains. Journal of Research in Personality, 37(6):504528, December 2003.

9. OP Oliver P John and Sanjay Srivastava. The Big Five trait taxonomy: History, measurement, and theoretical perspectives. In Lawrence A Pervin and Oliver P John, editors, Handbook of personality: Theory and research, volume 2, pages 102138. Guilford Press, New York, second edition, 1999.

10. M. Kosinski, D. Stillwell, and T. Graepel. Private traits and attributes are predictable from digital records of human behavior. Proceedings of the National Academy of Sciences, pages 2-5, March 2013.

11. Cynthia C S Liem and Ron Van Der Sterren. Innovating the Classical Music Experience in the PHENICX Project : Use Cases and Initial User Feedback. 1st International Workshop on Interactive Content Consumption (WSICC) at EuroITV 2013, 2013.

12. Daniel Müllensiefen, Bruno Gingras, Jason Musil, and Lauren Stewart. The musicality of non-musicians: an index for assessing musical sophistication in the general population. PloS one, 9(2):e89642, January 2014.

13. Daniele Quercia, Michal Kosinski, David Stillwell, and Jon Crowcroft. Our Twitter Profiles, Our Selves: Predicting Personality with Twitter. In 2011 SocialCom and PASSAT conference Proceedings, pages 180-185. IEEE, October 2011.

14. Peter J. Rentfrow and Samuel D. Gosling. The do re mi's of everyday life: The structure and personality correlates of music preferences. Journal of Personality and Social Psychology, 84(6):1236-1256, 2003.

15. M Tkalcic, M Kunaver, A Košir, and J Tasic. Addressing the new user problem with a personality based user similarity measure. Proceedings of the DEMRA 2011 and the UMMS 2011 Workshops, Girona, Spain, 2011. 